\title{
CORRECTION
}

\section{Author Correction: Cis-stilbene glucoside in Polygonum multiflorum induces immunological idiosyncratic hepatotoxicity in LPS-treated rats by suppressing PPAR- $\gamma$}

Ya-kun Meng ${ }^{1,2}$, Chun-yu Li ${ }^{1,3}$, Rui-yu Li ${ }^{1}$, Lan-zhi He ${ }^{1}$, He-rong Cui ${ }^{1}$, Ping Yin ${ }^{1}$, Cong-en Zhang ${ }^{1}$, Peng-yan Li ${ }^{1}$, Xiu-xiu Sang ${ }^{1}$, Ya Wang ${ }^{1}$, Ming Niu', Ya-ming Zhang ${ }^{1}$, Yu-ming Guo ${ }^{1}$, Rong Sun ${ }^{4}$, Jia-bo Wang ${ }^{1}$, Zhao-fang Bai ${ }^{1}$ and Xiao-he Xiao ${ }^{1}$

Acta Pharmacologica Sinica (2021) 42:1723-1724; https://doi.org/10.1038/s41401-020-00592-2

Correction to: Acta Pharmacol Sin https://doi.org/10.1038/ aps.2017.32, published online 26 June 2017

The authors regret that there were some immunohistochemical figures errors in Fig. $2 \mathrm{C}$ and Fig. $6 \mathrm{D}$ owing to the mistakes of copying and pasting in the process of assembling figures. In Fig. 2C, the "Trans-SG" group of Ki67 staining is repeated with "LPS + Trans-SG" group in Fig. 2C. In Fig. 6D, the "LPS + Cis-SG + Pioglitazone" group of TUNEL staining is repeated with "Trans-SG" group in Fig. 2D. The "LPS" group of TUNEL staining in Fig. 2D is repeated with "LPS" group in Fig. 6D. All the authors agree on the correction of our negligence, and the correct Fig. 2C "Trans-SG", Fig. 6D "LPS + Cis-SG + Pioglitazone" and "LPS" groups are provided. The revisions do not affect any results and conclusions of the current work.

The authors apologize for any inconvenience caused to the journal and readers.

Fig. $2 \mathrm{C}$ and Fig. $6 \mathrm{D}$ are revised as
$\mathrm{C}$

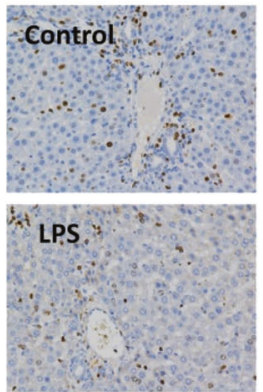

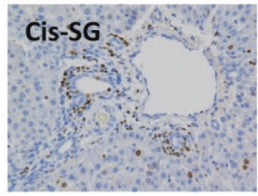

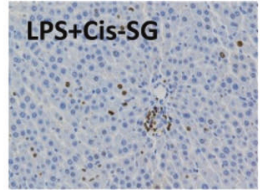

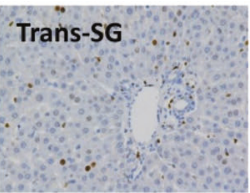

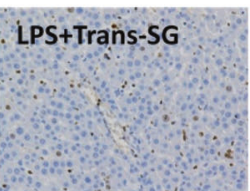

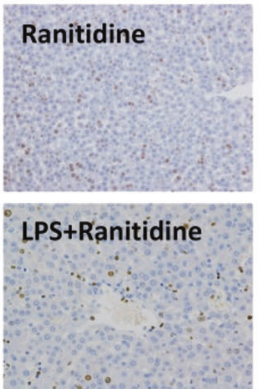

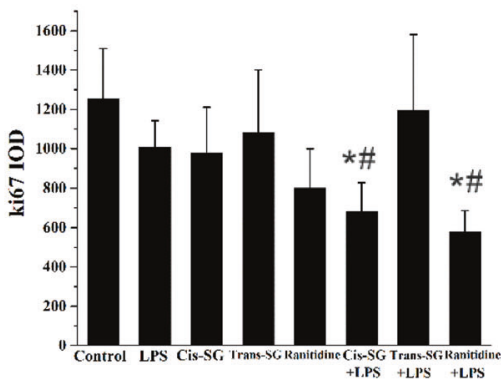

Fig. 2 (C) Representative micrographs of positive Ki67 staining (indicative of cell proliferation) in liver tissues obtained from rats treated with different conditions (200x). The integral optical density (IOD) of Ki67 was calculated by Image-Pro Plus 6.0. The cellular apoptosis rate was calculated by using Image-Pro Plus 6.0 and is represented as the apoptosis rate\%. The results are represented as mean \pm SEM $(n=8) .{ }^{*} P<0.05$ vs control. ${ }^{\#} P<0.05$ vs LPS as determined by ANOVA

\footnotetext{
${ }^{1}$ China Military Institute of Chinese Medicine, 302 Military Hospital, Beijing 100039, China; ${ }^{2}$ Clinic Trial Department, Leadingpharm Medical Technology, Inc, Beijing 100083, China; ${ }^{3}$ Institute of Medicinal Plant Development, Chinese Academy of Medical Sciences \& Peking Union Medical College, Beijing 100193, China and ${ }^{4}$ Shandong Academy of Chinese Medicine, Ji'nan 250014, China

Correspondence: Jia-bo Wang (wjb0128@126.com) or Zhao-fang Bai (baizf2008@126.com) or Xiao-he Xiao (pharmacy302xxh@163.com)
}

Published online: 22 December 2020 
$\mathrm{D}$
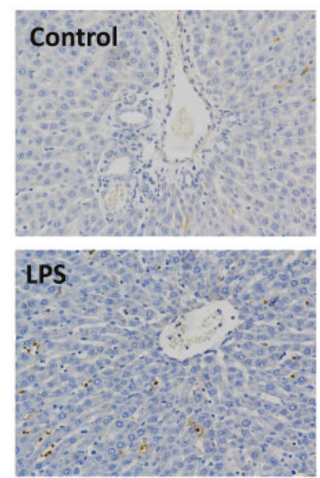
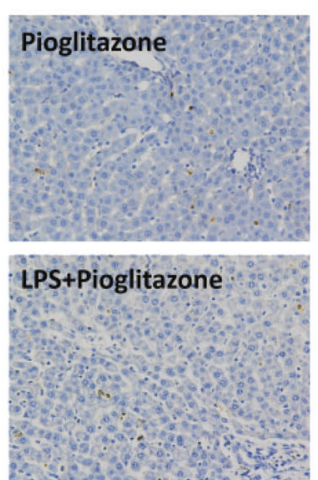
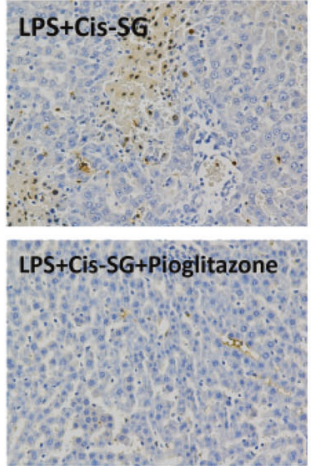

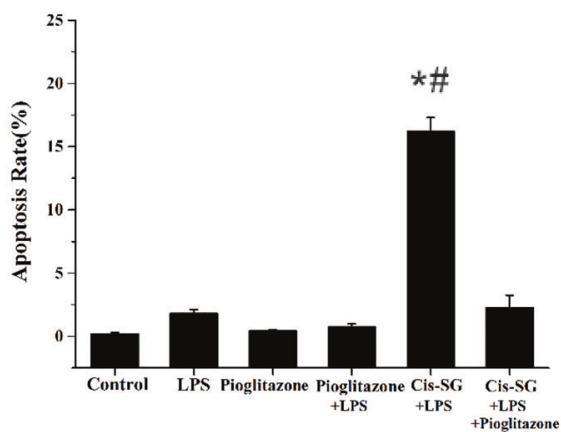

Fig. 6 (D) Representative micrographs of positive TUNEL staining (indicative of cell apoptosis) in liver tissues obtained from rats subjected to different treatment conditions. The cellular apoptosis rate was calculated by Image-Pro Plus 6.0 and represented as apoptosis rate. The results are represented as mean \pm SEM. $n=8$. ${ }^{*} P<0.05$ vs LPS. ${ }^{\#} P<0.05$ vs LPS+cis-SG+pioglitazone as determined by ANOVA 